\section{Avifaunal diversity in the University Campus of Kurukshetra, Haryana}

\section{Sanjeev K. Gupta, Parmesh Kumar \& Manoj Kumar Malik}

Department of Zoology, University College, Kurukshetra University, Kurukshetra, Haryana 136119, India

Email: sanjeevkuk@gmail.com

Birds are common denizens of the ecosystem and are considered indicator species (Blair 1999). They serve as monitors to examine the long term effects of habitat fragmentation. The Indian subcontinent has 1340 bird species (Ali \& Ripley 1987; Manakadan \& Pittie 2001). Recent developmental activities in the urban areas have led to large scale reduction in the quality of natural habitats. This has prompted us to make a survey of birds in Kurukshetra university campus.

Study Area: Kurukshetra University $\left(29^{\circ} 57^{\prime} 44.62^{\prime \prime}\right.$ N \& $76^{\circ} 49^{\prime} 10.80^{\prime \prime} \mathrm{E}$ ) is located in Haryana, at a distance of $4 \mathrm{~km}$. from Kurukshetra Railway Station and $8 \mathrm{kms}$. from Pipli. Kurukshetra is very hot in summer and remarkably cold in winter, with atmospheric temperature varying from $45^{\circ} \mathrm{C}$ in summer and $2^{\circ} \mathrm{C}$ in winter. The 160 ha university campus is situated on the western bank of the holy tank, Brahm Sarovar. A channel connecting Bhakra canal with Brahm Sarovar passes through the campus. The campus is surrounded by agricultural lands where wheat and paddy are cultivated. The important vegetation of the campus includes trees like Mangifera indica, Syzigium cumini, Eucalyptus hybrida, Syzigium frutcience, Morus alba, Citrus jambhiri, Eriobotrya japonica, Psydium quajava, Ficus carica, Azadirachta indica, Alstonia scholaris, Ficus bengalensis, Tectona grandis, Aegle marmelos, Emblica officinalis, Polyaithia longifolia, Thuja compacta, Delonix regia, Plumeria acutifolia, Terminalia arjuna, Bauhinia variegata, Kigelia sp., Ficus religiosa, Cassia fistula, Populus sp., Grevillea robusta, Mimusops elengi etc. The dominant shrubs are Cassia javanica, Ervatamia coronaria,

Date of publication (online): 26 December 2009

Date of publication (print): 26 December 2009

ISSN 0974-7907 (online) | 0974-7893 (print)

Editor: J. Praveen

Manuscript details:

Ms \# 02159

Received 16 March 2009

Final received 19 October 2009

Finally accepted 01 December 2009

Citation: Gupta, S.K., P. Kumar \& M.K. Malik (2009). Avifaunal diversity in the University Campus of Kurukshetra, Haryana. Journal of Threatened Taxa 1(12): 629-632.

Copyright: (c) Sanjeev K. Gupta, Parmesh Kumar \& Manoj Kumar Malik 2009. Creative Commons Attribution 3.0 Unported License. JoTT allows unrestricted use of this article in any medium for non-profit purposes, reproduction and distribution by providing adequate credit to the authors and the source of publication.

Acknowledgments: We are grateful to Prof. D Mohan, IFS, Wild Life Institute, Dehradun, India for suggestions in identification of bird species.: to Dr. K.B. Singh, Lecturer in Botany, University College Kurukshetra for identification of vegetation in university campus.

OPEN AGGESS | FREE DOWNLOAD

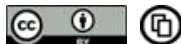

Bougainvillea sp., Thevetia peruviana, Murraya exotica, Hibiscusrosa sinensis, Lagerstromea parviflora, Hamelia patens, Zizyphus jujuba, Zizyphus numularia, Acacia arabica, Capparis deciduas, Jasminum sp., Calotropis procera, Euphorbia sp., Nerium oleander, Lantana chamaera, Clerodendron sp. etc. while Parthenium, Amaranthus spinosus, Chenopodium ambrosiodes, Canbis sativa are the prominent weeds.

Methods: The study was carried out in the campus for four years, i.e., January 2005 to December 2008. Regular surveys were done by walking on fixed routes through the study area. Birds were observed from 0600 to $1000 \mathrm{hr}$ and from 1600 to $1800 \mathrm{hr}$ and details on habitat type, season, feeding habit and status (resident/migrant, breeding/nonbreeding) and frequency of occurrence were recorded. Ali \& Ripley (1987) and Grimmet et al. (1999) were followed for identification. Breeding status was assigned after observing a standard protocol. The check list was prepared following Manakadan \& Pittie (2001). Occurrence criteria were, Common recorded 910 times out of 10 visits, Fairly common recorded 6 -8 times out of 10 visits, Uncommon recorded $3-5$ times out of 10 visits, Rare recorded $\mathrm{O}-2$ times out of 10 visits, and Irregular.

Results and Discussion: A total of 92 bird species belonging to 67 genera and 37 families were recorded which comprised 46 non passerine and 46 passerine species (Table 1). In this study Passeriformes was dominant with 18 families. Of all, family Turdinae dominated with 07 species. Out of total 92 species, 71 were resident and 21 were migrant species. Most of the migratory species were winter visitors except Pied Crested Cuckoo, Brainfever Bird and Eurasian Golden Oriole which were summer visitors. Out of 71 resident species, 60 species have been observed to be positively breeding in the campus. Grey Francolin, Rose-ringed Parakeet, Spotted Owlet, Blue Rock Pigeon, Spotted Dove, Red-wattled Lapwing, House Crow, Black Drongo, Common Myna, Jungle Babbler, Purple

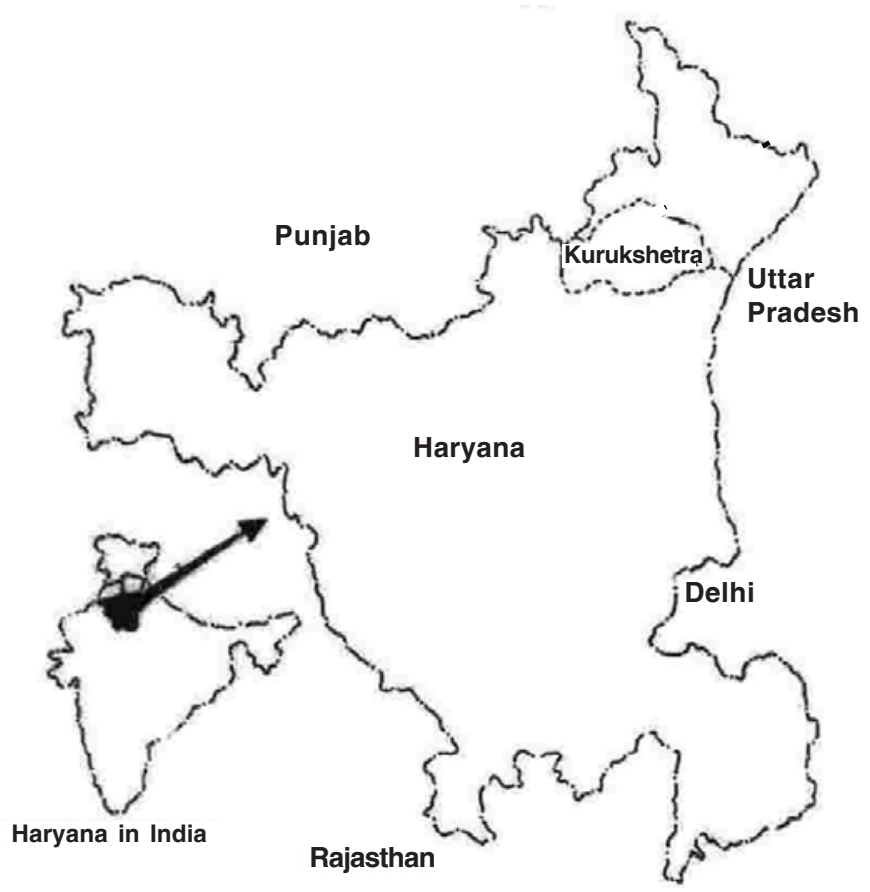

Figure 1. Location of the study area 
Table 1. List of birds recorded in the university campus of Kurukshetra

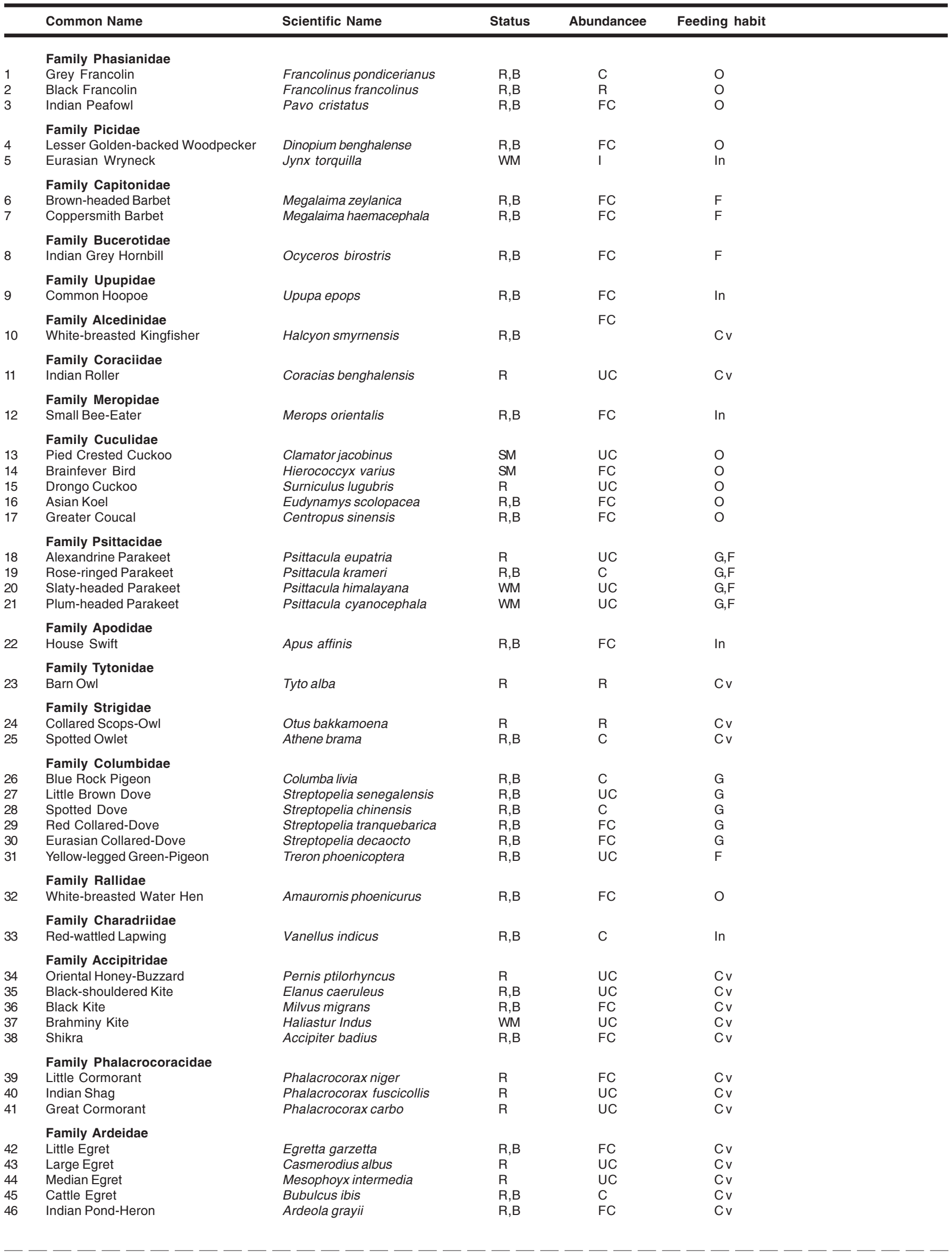




\begin{tabular}{|c|c|c|c|c|c|}
\hline & Common Name & Scientific Name & Status & Abundancee & Feeding habit \\
\hline & Family Lannidae & & & & \\
\hline 47 & Bay-backed Shrike & Lanius vittatus & $\mathrm{R}, \mathrm{B}$ & UC & $\mathrm{Cv}$ \\
\hline 48 & Rufous-backed Shrike & Lanius schach & $R, B$ & UC & $\mathrm{Cv}$ \\
\hline & Family Corvidae & & & $\mathrm{FC}$ & $\mathrm{O}$ \\
\hline 49 & Indian Treepie & Dendrocitta vagabunda & $\mathrm{R}, \mathrm{B}$ & & \\
\hline 50 & House Crow & Corvus splendens & $\mathrm{R}, \mathrm{B}$ & C & $\mathrm{O}$ \\
\hline 51 & Jungle Crow & Corvus macrorhynchos & WM & UC & $\mathrm{O}$ \\
\hline $\begin{array}{l}52 \\
53\end{array}$ & $\begin{array}{l}\text { Family Campephagidae } \\
\text { Long-tailed Minivet } \\
\text { Small Minivet }\end{array}$ & $\begin{array}{l}\text { Pericrocotus ethologus } \\
\text { Pericrocotus cinnamomeus }\end{array}$ & $\begin{array}{l}\text { WM } \\
\text { WM }\end{array}$ & $\begin{array}{l}\text { FC } \\
\text { UC }\end{array}$ & $\begin{array}{l}\text { In } \\
\ln \end{array}$ \\
\hline 54 & $\begin{array}{l}\text { Family Monarchinae } \\
\text { Asian Paradise-Flycatcher }\end{array}$ & Terpsiphone paradisi & WM & $\mathrm{R}$ & In \\
\hline 55 & $\begin{array}{l}\text { Family Rhipidurinae } \\
\text { White-browed Fantail-Flycatcher }\end{array}$ & Rhipidura aureola & WM & $\mathrm{FC}$ & $\ln$ \\
\hline 56 & $\begin{array}{l}\text { Family Dicruridae } \\
\text { Black Drongo }\end{array}$ & Dicrurus macrocercus & $\mathrm{R}, \mathrm{B}$ & C & $\mathrm{O}$ \\
\hline $\begin{array}{l}57 \\
58 \\
59 \\
60 \\
61 \\
62 \\
63\end{array}$ & $\begin{array}{l}\text { Family Turdinae } \\
\text { Dark-Throated Thrush } \\
\text { Bluethroat } \\
\text { Oriental Magpie Robin } \\
\text { Indian Robin } \\
\text { Common Stonechat } \\
\text { Pied Bushchat } \\
\text { Indian Chat }\end{array}$ & $\begin{array}{l}\text { Turdus ruficollis } \\
\text { Luscinia svecica } \\
\text { Copsychus saularis } \\
\text { Saxicoloides fulicata } \\
\text { Saxicola torquata } \\
\text { Saxicola caprata } \\
\text { Cercomela fusca }\end{array}$ & $\begin{array}{l}\text { WM } \\
\text { WM } \\
\text { R,B } \\
\text { R,B } \\
\text { WM } \\
\text { R,B } \\
\text { R,B }\end{array}$ & $\begin{array}{l}\text { I } \\
\text { FC } \\
\text { FC } \\
\text { FC } \\
\text { FC } \\
\text { UC }\end{array}$ & $\begin{array}{l}\text { In } \\
\text { In } \\
\text { O } \\
\text { In } \\
\text { In } \\
\text { In } \\
\text { In }\end{array}$ \\
\hline $\begin{array}{l}64 \\
65 \\
66 \\
67 \\
68\end{array}$ & $\begin{array}{l}\text { Family Sturnidae } \\
\text { Brahminy Starling } \\
\text { Asian Pied Starling } \\
\text { Common Myna } \\
\text { Bank Myna } \\
\text { Jungle Myna }\end{array}$ & $\begin{array}{l}\text { Sturnus pagodarum } \\
\text { Sturnus contra } \\
\text { Acridotheres tristis } \\
\text { Acridotheres ginginianus } \\
\text { Acridotheres fuscus }\end{array}$ & $\begin{array}{l}R, B \\
R, B \\
R, B \\
R, B \\
\text { WM }\end{array}$ & $\begin{array}{l}\text { FC } \\
\text { FC } \\
\text { C } \\
\text { FC } \\
\text { UC }\end{array}$ & $\begin{array}{l}0 \\
0 \\
0 \\
0 \\
0\end{array}$ \\
\hline 69 & $\begin{array}{l}\text { Family Oriolidae } \\
\text { Eurasian Golden Oriole }\end{array}$ & Oriolus oriolus & SM & FC & $\mathrm{O}$ \\
\hline $\begin{array}{l}70 \\
71 \\
72\end{array}$ & $\begin{array}{l}\text { Family Hirundinidae } \\
\text { Plain Martin } \\
\text { Common Swallow } \\
\text { Wire-tailed Swallow }\end{array}$ & $\begin{array}{l}\text { Riparia paludicola } \\
\text { Hirundo rustica } \\
\text { Hirundo smithii }\end{array}$ & $\begin{array}{l}R, B \\
R, B \\
R, B\end{array}$ & $\begin{array}{l}\mathrm{FC} \\
\mathrm{FC} \\
\mathrm{FC}\end{array}$ & $\begin{array}{l}\ln \\
\ln \\
\ln \end{array}$ \\
\hline 73 & $\begin{array}{l}\text { Family Pycnonotidae } \\
\text { Red-vented Bulbul }\end{array}$ & Pycnonotus cafer & $\mathrm{R}, \mathrm{B}$ & $\mathrm{C}$ & $\ln$ \\
\hline 74 & $\begin{array}{l}\text { Family Zosteropidae } \\
\text { Oriental White-Eye }\end{array}$ & Zosterops palpebrosus & $R, B$ & UC & $\mathrm{O}$ \\
\hline $\begin{array}{l}75 \\
76 \\
77 \\
78 \\
79\end{array}$ & $\begin{array}{l}\text { Family Sylviinae } \\
\text { Ashy Prinia } \\
\text { Common Lesser Whitethroat } \\
\text { Paddyfield Warbler } \\
\text { Common Chiffchaff } \\
\text { Common Tailorbird }\end{array}$ & $\begin{array}{l}\text { Prinia socialis } \\
\text { Sylvia curruca } \\
\text { Acrocephalus agricola } \\
\text { Phylloscopus collybita } \\
\text { Orthotomus sutorius }\end{array}$ & $\begin{array}{l}R, B \\
\text { WM } \\
R, B \\
\text { WM } \\
R, B\end{array}$ & $\begin{array}{l}\text { FC } \\
\text { FC } \\
\text { UC } \\
\text { FC } \\
\text { FC }\end{array}$ & $\begin{array}{l}\text { In } \\
\text { In } \\
\text { In } \\
\text { In } \\
\text { In,N }\end{array}$ \\
\hline $\begin{array}{l}80 \\
81 \\
82\end{array}$ & $\begin{array}{l}\text { Family Timaliinae } \\
\text { Common Babbler } \\
\text { Large Grey Babbler } \\
\text { Jungle Babbler }\end{array}$ & $\begin{array}{l}\text { Turdoides caudatus } \\
\text { Turdoides malcolmi } \\
\text { Turdoides striatus }\end{array}$ & $\begin{array}{l}R, B \\
R, B \\
R, B\end{array}$ & $\begin{array}{l}\mathrm{FC} \\
\mathrm{FC} \\
\mathrm{C}\end{array}$ & $\begin{array}{l}\mathrm{O} \\
\mathrm{O} \\
\mathrm{O}\end{array}$ \\
\hline 83 & $\begin{array}{l}\text { Family Nectariniidae } \\
\text { Purple Sunbird }\end{array}$ & Nectarinia asiatica & $\mathrm{R}, \mathrm{B}$ & C & $\ln , \mathrm{N}$ \\
\hline $\begin{array}{l}84 \\
85 \\
86 \\
87 \\
88\end{array}$ & $\begin{array}{l}\text { Family Motacillidae } \\
\text { White Wagtail } \\
\text { Large Pied Wagtail } \\
\text { Citrine Wagtail } \\
\text { Yellow Wagtail } \\
\text { Paddyfield Pipit }\end{array}$ & $\begin{array}{l}\text { Motacilla alba } \\
\text { Motacilla maderaspatensis } \\
\text { Motacilla citreola } \\
\text { Motacilla flava } \\
\text { Anthus rufulus }\end{array}$ & $\begin{array}{l}\text { WM } \\
\text { R,B } \\
\text { WM } \\
\text { WM } \\
\text { R,B }\end{array}$ & $\begin{array}{l}\text { FC } \\
\text { FC } \\
\text { UC } \\
\text { UC } \\
\text { UC }\end{array}$ & $\begin{array}{l}\text { In } \\
\text { In } \\
\text { In } \\
\text { In } \\
\text { In }\end{array}$ \\
\hline $\begin{array}{l}89 \\
90\end{array}$ & $\begin{array}{l}\text { Family Ploceinae } \\
\text { Baya Weaver } \\
\text { Streaked Weaver }\end{array}$ & $\begin{array}{l}\text { Ploceus philippinus } \\
\text { Ploceus manyar }\end{array}$ & $\begin{array}{l}\mathrm{R}, \mathrm{B} \\
\mathrm{R}, \mathrm{B}\end{array}$ & $\begin{array}{l}\text { UC } \\
\text { UC }\end{array}$ & $\begin{array}{l}\mathrm{I}, \mathrm{G} \\
\mathrm{I}, \mathrm{G}\end{array}$ \\
\hline $\begin{array}{l}91 \\
92\end{array}$ & $\begin{array}{l}\text { Family Estrildidae } \\
\text { White-throated Munia } \\
\text { Spotted Munia }\end{array}$ & $\begin{array}{l}\text { Lonchura malabarica } \\
\text { Lonchura punctulata }\end{array}$ & $\begin{array}{l}\mathrm{R}, \mathrm{B} \\
\mathrm{R}, \mathrm{B}\end{array}$ & $\begin{array}{l}\text { FC } \\
\text { FC }\end{array}$ & $\begin{array}{l}\mathrm{G} \\
\mathrm{I}, \mathrm{G}\end{array}$ \\
\hline
\end{tabular}

R - Resident; SM - Summer migrant; WM - Winter migrant; B - Breeding; C - Common; FC - Fairly common; UC - Uncommon; R - Rare; I - Irregular; In Insectivore; O - Omnivore; Cv - Carnivore; G - Granivore; F - Fruigivore; N - Nectarivore. 
Sunbird and Red Vented Bulbul were common residents. Eurasian Wryneck and Dark-throated Thrush were irregular visitors. Surprisingly the House Sparrow (Passer domesticus), a very common breeding resident, could not be recorded even once in the university campus during the entire span of the survey. The campus avifauna is composed of insectivores (33) followed by omnivores (25), carnivores (20), granivores (13), fruigivores (8) and nectarivores (2). The campus calls for more efforts in biodiversity and ecology conservation.

\section{References}

Ali, S. \& S.D. Ripley (1987). Compact Handbook of the Birds of India and Pakistan together with those of Bangladesh, Nepal, Bhutan and Sri Lanka. Oxford University Press, Delhi, India, 737pp.

Blair, R.B. (1999). Birds and butterflies; surrogate taxa for assessing biodiversity? Ecolological Application 9: 164-170.

Grimmett, R., C. Inskipp \& T. Inskipp (1999). Pocket guide to the birds of the Indian subcontinent. Oxford University Press, Delhi, India, 384pp.

Manakadan, R. \& A. Pittie (2001). Standardised common and scientific names of the birds of the Indian subcontinent. Buceros-Envis Newsletter $6(1): 1-37$ 